\title{
Granzyme B in Atherosclerosis and Transplant Vascular Disease: Association with Cell Death and Atherosclerotic Disease Severity
}

Jonathan C. Choy, B.Sc., Paul C. McDonald, Ph.D., Agripina C. Suarez, B.Sc., Vivian H. Y. Hung, Janet E. Wilson, B.Sc., M.T., Bruce M. McManus, M.D., Ph.D., David J. Granville, Ph.D.

The iCAPTUR ${ }^{4}$ E Centre/UBC McDonald Research Laboratories, Department of Pathology and Laboratory Medicine, St. Paul's Hospital/Providence Health Care-University of British Columbia, Vancouver, British Columbia, Canada

Apoptosis of intimal cells is an important contributor to the pathogenesis of atherosclerosis and transplant vascular disease (TVD). Since the activated immune response may be a key regulator of apoptosis in these lesions, we used immunohistochemistry to characterize the presence and localization of granzyme B, a major mediator of the cytotoxic immune response, in advanced atherosclerosis and TVD. Formalin-fixed, paraffin-embedded transverse sections from human left anterior descending coronary arteries were cut serially and stained with antibodies specific for granzyme B, smooth muscle $\alpha$-actin, CD68, and CD3. The amount of granzyme B staining was semi-quantitated on a $0-5+/ 5+$ scale. Also, TUNEL staining and in situ hybridization was performed to visualize cells undergoing cellular damage suggestive of apoptosis, and to localize granzyme B mRNA, respectively. Granzyme B localization was similar in both diseases. This protease was absent in arteries with mild atherosclerosis, but was abundant in the intima and media of vessels with advanced atherosclerosis and TVD. Within the intima, granzyme B localized to TUNEL-positive foam cells surrounding lipid-rich atheromas. Staining of serial sections with granzyme $B$ and either

\footnotetext{
Copyright (C) 2003 by The United States and Canadian Academy of Pathology, Inc.

VOL. 16, NO. 5, P. 460, 2003 Printed in the U.S.A.

Date of acceptance: February 13, 2003.

This work was supported in part by the St. Paul's Hospital Foundation, grant-in-aid from the Heart and Stroke Foundation of British Columbia and Yukon, an operating grant from the Canadian Institutes of Health Research (BMM), a Personnel Traineeship from the Heart and Stroke Foundation of British Columbia and Yukon (PCM), and Doctoral Research Awards funded by the Michael Smith Foundation for Health Research, Canadian Institutes of Health Research and the Estate of Dorothy and Izaak Walton Killam (JCC). DJG is a recipient of a Canada Research Chair in Cardiovascular Biochemistry.

Address reprint requests to: David J. Granville, Ph.D., The iCAPTUR ${ }^{4} E$ Centre/UBC McDonald Research Laboratories, Room 292, Burrard Building, St. Paul's Hospital, Vancouver, British Columbia, V6Z 1Y6 Canada; fax: (604) 806-8351; e-mail: dgranville@mrl.ubc.ca.

DOI: 10.1097/01.MP.0000067424.12280.BC
}

smooth muscle $\alpha$-actin, anti-CD68, or anti-CD3 showed that granzyme B localized to smooth muscle cells, macrophages, and T-cells. Further, in situ hybridization for granzyme B mRNA in TVD cases localized its expression to infiltrating leukocytes and not foam cells. In conclusion, the presence of granzyme $B$ in advanced atherosclerotic lesions and TVD is associated with increasing disease severity and cell death. These observations suggest that granzyme B-mediated apoptosis may contribute to the pathogenesis of these diseases.

KEY WORDS: Apoptosis, Atherosclerosis, Granzyme B, Macrophage, Smooth muscle cell, Transplant vascular disease.

Mod Pathol 2003;16(5):460-470

Apoptosis is a key contributor to the pathogenesis of inflammatory vascular disorders such as atherosclerosis and transplant vascular disease (TVD). The role of smooth muscle cell (SMC) and macrophage apoptosis in atherosclerosis may depend on the type of lesion affected. In early, stable lesions, increased SMC apoptosis may be a beneficial way to decrease intimal hyperplasia (1). However, in advanced, unstable lesions, increased SMC apoptosis decreases the cellularity of affected lesions, thereby compromising the synthesis of extracellular matrix components and decreasing plaque stability $(2,3)$. Although the role of macrophage apoptosis is less well defined, an increase in this event may promote atherosclerosis by reducing the ability of these phagocytes to effectively clear lipid deposits from the atheromatous core, or by promoting the release of matrix-degrading enzymes.

TVD is an immune-triggered accelerated inflammatory disorder that affects the vasculature of solid organ allografts. Apoptosis of the endothelium and smooth muscle cells is probably a key contributor to TVD. Because the endothelium is crucial to homeostatic maintenance of the entire vasculature, 
increased endothelial cell apoptosis could promote vascular disease by increasing smooth muscle migration and proliferation, vascular permeability to leukocytes and circulating lipids, and platelet activation (4). Indeed, endothelial damage is observed early during TVD and is likely initiated by alloimmune attack by $\mathrm{T}$ cells (5). Although the role and inducers of SMC apoptosis in TVD is not clear, increased apoptosis of this cell type could either decrease intimal thickening in the early stages of this disease or promote lipid accumulation in advanced lesions.

In both atherosclerosis and TVD, the activated immune response is believed to be a key inducer of apoptosis. Both CD4+ and CD8+ T cells accumulate in atherosclerotic and TVD plaques, and there is an antigen-driven T-cell response in patients with unstable angina $(6,7)$. CD8 $+\mathrm{T}$ cells have been found to localize to the shoulder region of atherosclerotic plaques and have been well associated with plaque rupture, suggesting that the cytotoxic immune response plays a role in decreasing plaque stability (8). Immune cells can initiate apoptosis of target cells through FasL and granzyme B/perforin-mediated pathways. FasL binds to Fas and can promote the recruitment of adaptor proteins and caspase- 8 to the plasma membrane. These molecules form the deathinducing signaling complex (DISC), resulting in the activation of downstream caspases and apoptotic cell death (9). Secretory granules cytotoxic T-cells contain granzyme B and perforin (10). Granzyme B is a 32$\mathrm{kDa}$ cytotoxic serine protease. In the presence of perforin, it can enter the cytoplasm of cells and induce apoptosis by either direct or indirect activation of caspases. Granzyme B-mediated apoptosis is important in host defenses against viral infection or in response to foreign antigen inherent to allogeneic transplantation (11).

There is increased expression of FasL in unstable atherosclerotic plaques as compared with more stable, fibrous plaques (12). In TVD, increased expression of Fas by the endothelium and localization of Fas to apoptotic endothelial cells suggests that this type of immune-mediated cytotoxicity is an important contributor to vascular injury (13). However, the presence and role of granzyme B-mediated apoptosis in atherosclerosis and TVD is not clear. In this study we associate the presence of granzyme B in native atheromas with atherosclerotic disease severity and characterize the localization of this protease in atherosclerosis and TVD.

\section{METHODS}

\section{Cases and Tissue Processing}

Spleen and tonsil sections used for optimization of the granzyme B immunohistochemistry and in situ hybridization, respectively, were obtained from autopsy specimens at St. Paul's Hospital, Department of Pathology and Laboratory Medicine. Left anterior descending coronary arteries from 24 randomly selected patients from the St. Paul's Hospital Cardiovascular Registry were studied. Six patients had very early disease that was defined as a limited intimal cushion and a cellular intima. Eleven patients had advanced atherosclerotic lesions that were characterized by the presence of a large, lipidrich atheromatous core. Eight patients had moderate-severe TVD that was defined as arteries with a large, lipid-rich intima and $>50 \%$ crosssectional luminal narrowing. Patients in all groups were age comparable.

All samples were processed as reported elsewhere (14). Briefly, hearts were harvested as soon as possible after autopsy. Left anterior descending coronary arteries were then perfusion fixed with $10 \%$ neutral buffered formalin, removed intact from the heart, and sectioned transversely at 5- to $10-\mathrm{mm}$ intervals. Fixed sections were then embedded in paraffin.

\section{Immunohistochemistry}

Formalin-fixed, paraffin-embedded sections of proximal left anterior descending coronary arteries were sectioned serially $(3 \mu \mathrm{m})$. Slides were deparaffinized, and heat-based antigen retrieval was performed. Slides were incubated with $10 \%$ normal horse serum for 30 minutes, goat polyclonal antigranzyme B (Santa Cruz; Santa Cruz, CA) or monoclonal anti-CD68 (DAKO; Carpinteria, CA) for 1 hour, a 1:200 dilution of biotin horse anti-goat or biotin horse anti-mouse for 30 minutes, and alkaline phosphatase-conjugated avidin-biotin complex (DAKO) for 30 minutes at room temperature. Staining was visualized using the chromogen Vector Red (Vector Laboratories; Burlingame, CA), and hematoxylin was used as a nuclear counterstain.

Staining for smooth muscle $\alpha$-actin and CD3 was automated and performed on a Ventana immunostainer as described elsewhere (13). The sections were incubated with anti-smooth muscle $\alpha$-actin or anti-CD3 for 32 minutes, and with secondary antibody for 8 minutes at $37^{\circ} \mathrm{C}$. Staining was visualized with diaminobenzidine (3,3'-diaminobenzidine) and counterstained with hematoxylin.

\section{Western Blot}

Western blots on human coronary arteries were performed as described elsewhere $(15,16)$. Briefly, fresh frozen coronary arteries with advanced atherosclerosis and TVD were obtained from the St. Paul's Hospital Cardiovascular Registry. Samples were homogenized in $100 \mu \mathrm{L} / \mathrm{mg}$ of lysis buffer $/ \mathrm{mg}$ 


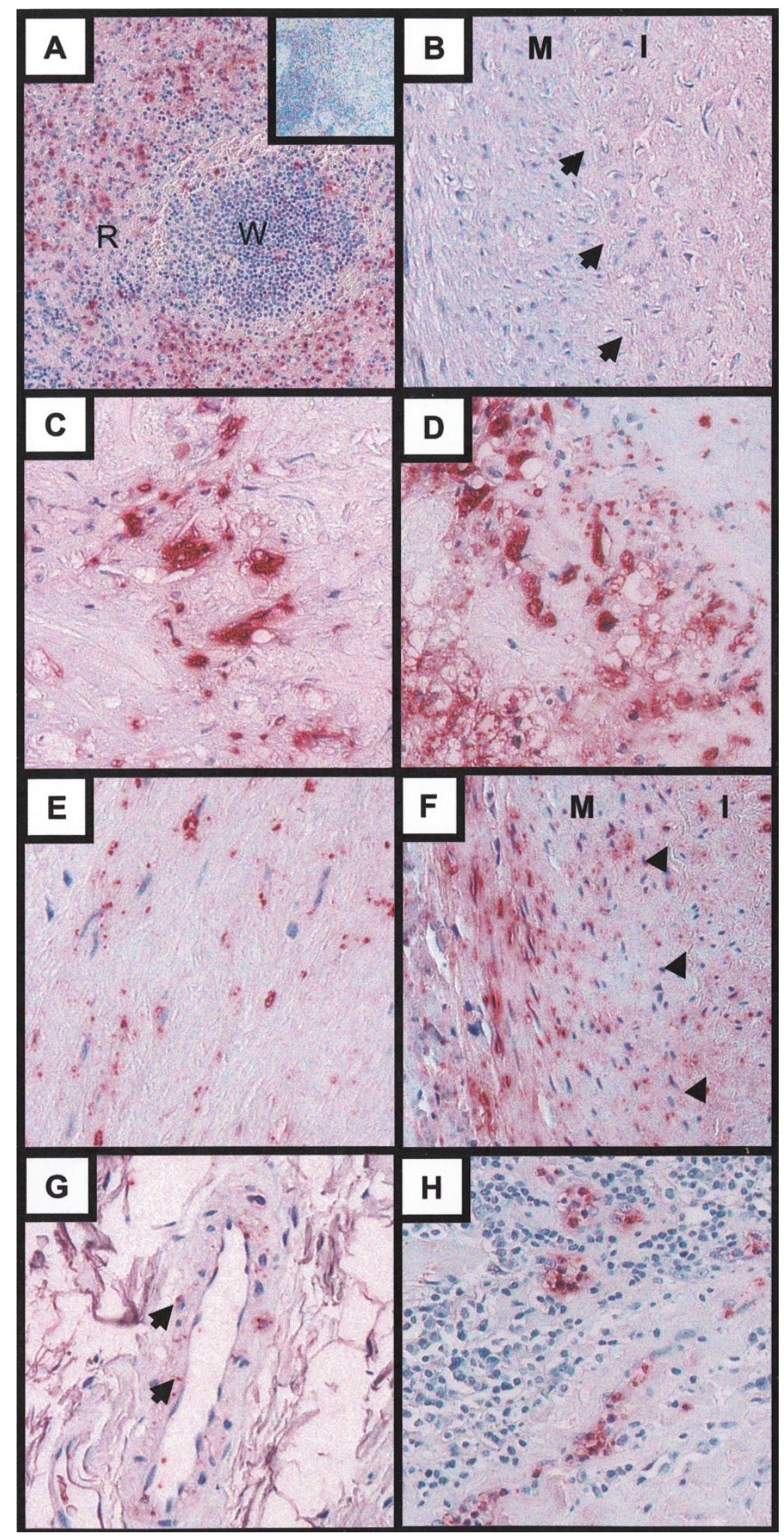

FIGURE 1. Granzyme B is present in advanced atherosclerosis and TVD. Human spleen sections were used as control tissue (A). Consistent with previous reports, granzyme B (red color) localizes to leukocytes in the red pulp of this organ but is absent from the white pulp ( $\mathrm{R}=$ red pulp, $\mathrm{W}=$ white pulp; $160 \times$ ). Addition of normal goat IgG in the place of the primary antibody resulted in negative staining (inset, $160 \times)$. In native vessels with a limited cushion (B), there is no granzyme B immunoreactivity (arrows = internal elastic lamina; $320 \times$ ). In the intima of vessels with advanced atherosclerosis (C) and TVD (D), granzyme B localizes to foam cells surrounding the lipid-rich atheroma. Granzyme B is also localized to the intimal shoulder regions of some vessels with advanced atherosclerosis $(320 \times)$. In the media of vessels with advanced atherosclerosis $(\mathbf{E})$, granzyme B immunoreactivity is very punctate, suggestive of its localization in $\mathrm{T}$ cells within this region of the vasculature $(320 \times)$. In the media of vessels with TVD $(\mathbf{F})$, granzyme B immunoreactivity is also very punctate but also appears to localize to some SMC (arrows, internal elastic lamina; $320 \times$ ). In the adventitia of vessels with advanced atherosclerosis (G), punctate granzyme B immunoreactivity is observed in some small adventitial arteries (arrows; $320 \times$ ). In this same region of vessels with TVD (H), granzyme B is observed in infiltrating perivascular leukocytes (320×). 
of tissue, centrifuged at 14,000 rpm for 10 minutes, and the supernatant was collected. Total protein concentration was quantified using a BCA protein assay kit (Pierce Chemicals; Rockford, IL), and 50 $\mu \mathrm{g}$ of total protein was run on a $10 \%$ polyacrylamide gel and transferred onto a nitrocellulose membrane. Granzyme B was detected using 2 $\mu \mathrm{g} / \mathrm{mL}$ of the goat polyclonal anti-granzyme B described above.

\section{In Situ TUNEL}

TUNEL staining was used to assess DNA fragmentation and was carried out as per the manufacturer's instructions (Intergen; Purchase, NY). Briefly, slides were deparaffinized, washed in PBS, incubated with $20 \mu \mathrm{g} / \mathrm{mL}$ of proteinase $\mathrm{K}$, and quenched in $3 \% \mathrm{H}_{2} \mathrm{O}_{2}$. Tissue sections were incubated with TdT enzyme for 1 hour at $37^{\circ} \mathrm{C}$ and then with HRP-conjugated anti-digoxigenin secondary antibody for 30 minutes at room temperature. Staining was visualized using 3,3'-diaminobenzidine, and hematoxylin was used as a nuclear counterstain.

\section{Histological Grading}

Qualitative and semiquantitative analysis was performed in a blinded fashion (by BMM). To assess reproducibility of semi-quantitative analysis, randomly selected slides were used to assess intraobserver variability on two separate occasions 15 days apart. A Student's $t$ test was used to determine statistical significance between vessel groups, with the $P$ value for significance set at .05 .

\section{In Situ Hybridization}

Granzyme B cDNA was a kind gift from Dr. R. Chris Bleackley (University of Alberta, Edmonton, Alberta, Canada) and was cloned into a pBlueScript-KS vector. Probes recognizing the full cDNA sequence of granzyme B were prepared using in vitro transcription. An irrelevant probe of the same length that recognizes coxsackievirus B3 RNA was synthesized in the same manner and used as a negative control.

In order to control for variations in mRNA levels that may result from differences in tissue processing, the localization and presence of total RNA was assessed in all arteries by staining with acridine/ orange. Most likely due to delayed fixation, atherosclerotic cases contained very low levels of RNA not suitable for in situ hybridization. However, arteries with TVD contained relatively abundant levels of RNA, and in situ hybridization was performed only on these tissues. The TVD cases were able to be harvested in an expedited manner as compared with autopsy-acquired atherosclerotic cases, and

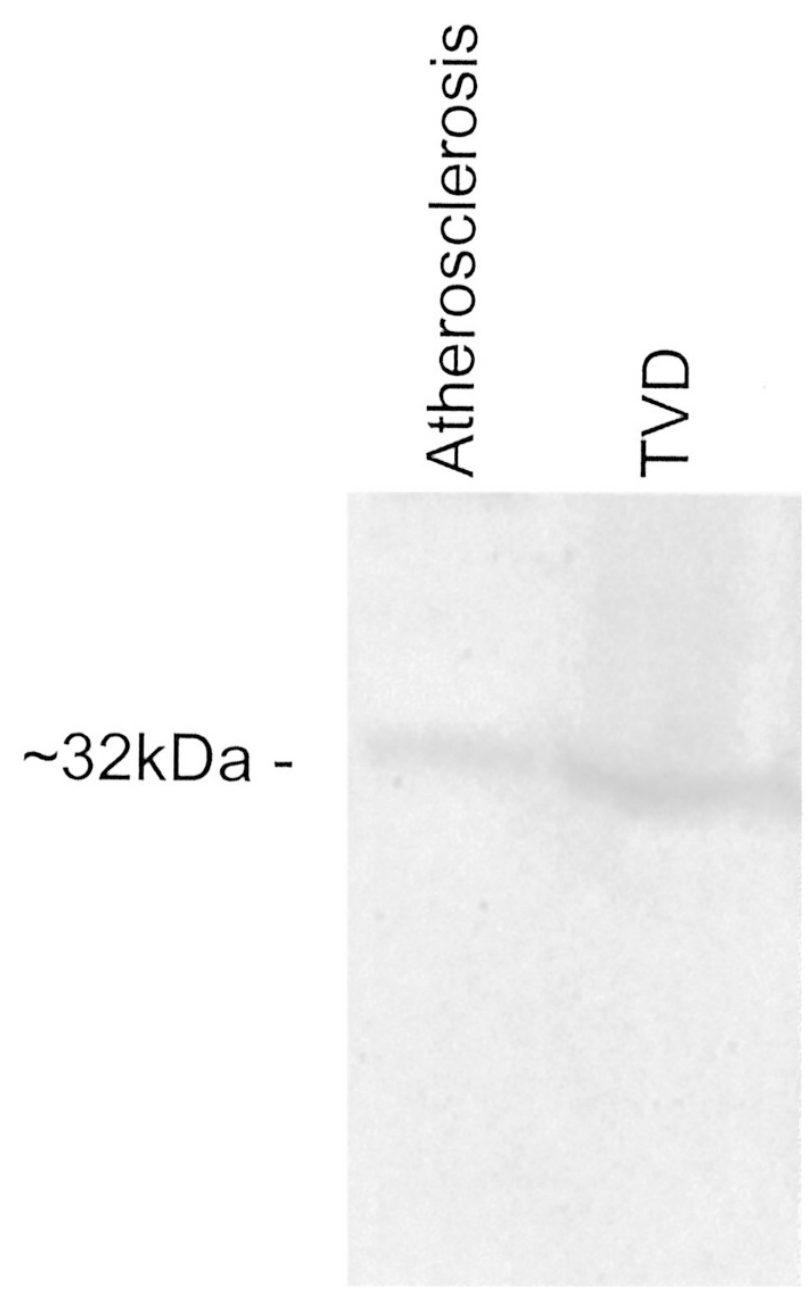

FIGURE 2. Western blot was performed on lysates from arteries with atherosclerosis and TVD. In both diseases, there is a single band corresponding to $\sim 32 \mathrm{kDa}$, indicating that granzyme B is abundant in both diseases and that the polyclonal anti-granzyme B used in the immunohistochemical staining is specific for this protease.

this difference in timing is the best explanation for the difference in RNA levels.

In situ hybridization was performed as described elsewhere (17). Briefly, paraffin-embedded tissue sections were dewaxed, incubated with proteinase $\mathrm{K}$, dehydrated, and hybridized with the appropriate probes. Hybridization was allowed to proceed at $42^{\circ}$ C overnight, followed by stringent washing in $50 \%$ formamide and $2 \times$ SSC. After hybridization, alkaline phosphatase-conjugated anti-digoxigenin antibody was applied to the tissue sections for $30 \mathrm{~min}$ utes and staining visualized with the color substrate Vector Red (Vector Laboratories).

\section{RESULTS}

Granzyme B is More Abundant in Advanced Atherosclerotic and TVD Lesions Than in Native Arteries with Mild Disease

Human spleen tissue was used as our control for optimization of immunohistochemistry for gran- 


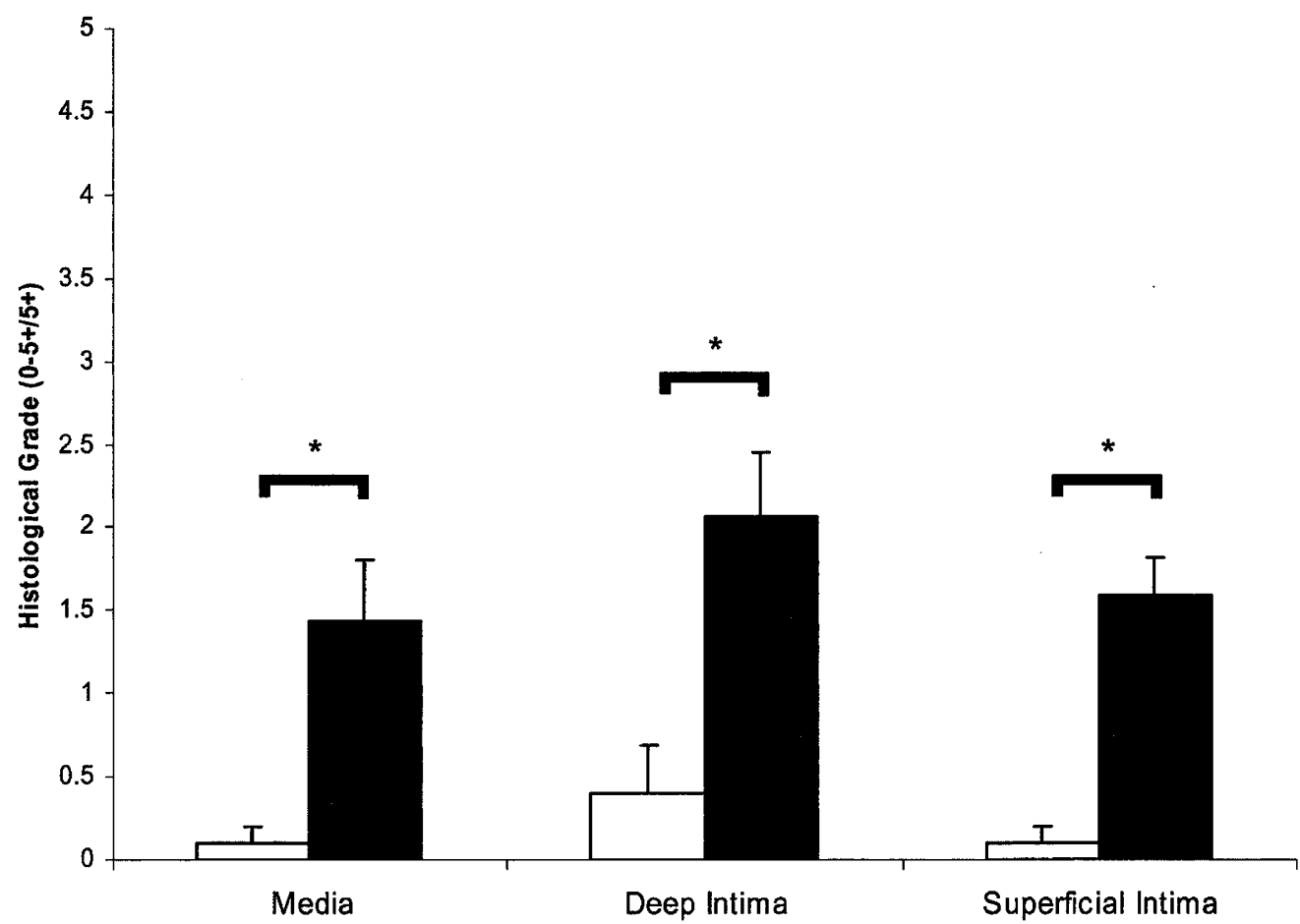

B

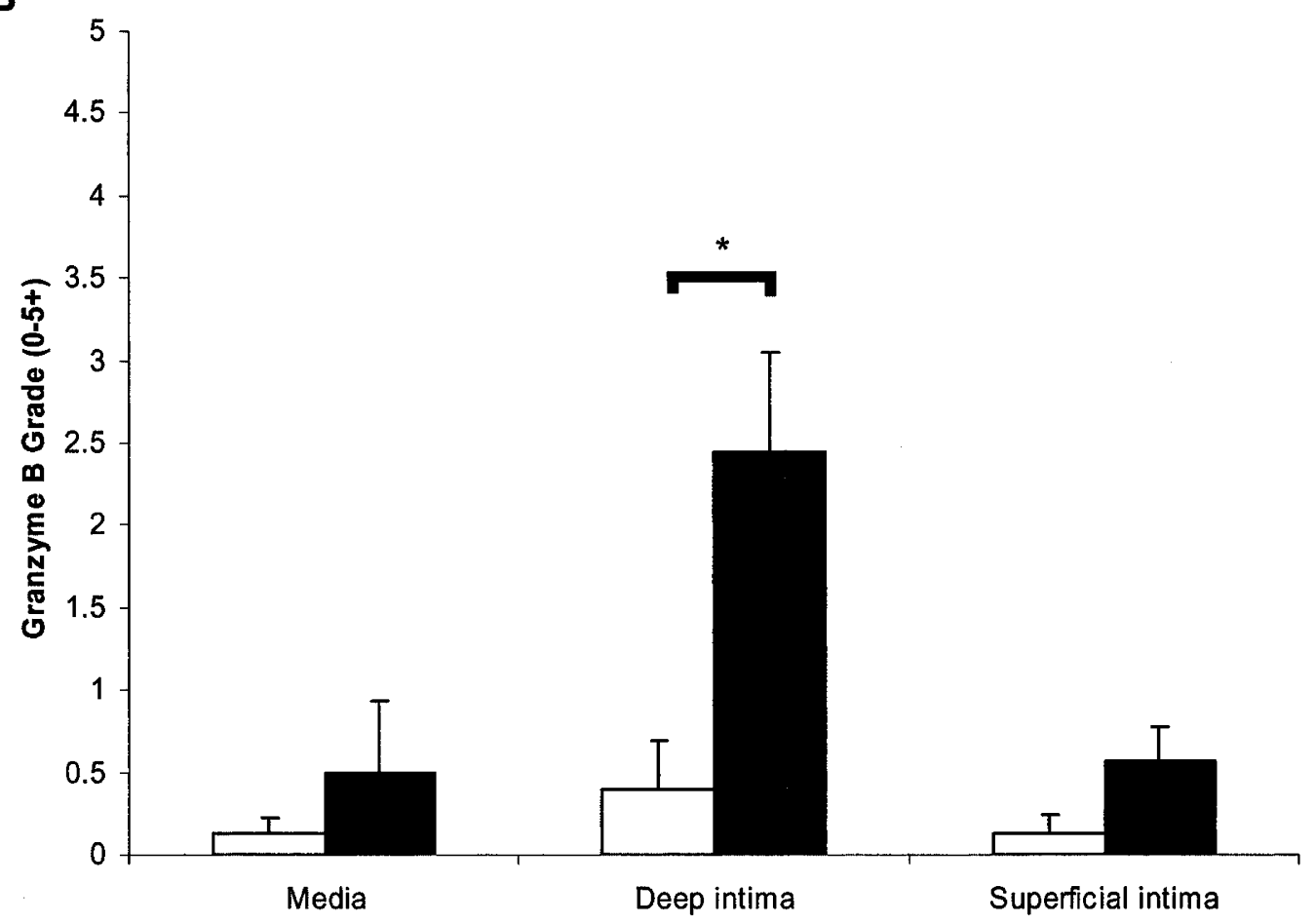

FIGURE 3. Semi-quantitative assessment of granzyme B immunoreactivity. Granzyme B immunoreactivity was scored on a $0-5+/ 5+$ scale. In advanced atherosclerotic lesions (A; black bars), there is significantly more granzyme B in the media, deep intima, and superficial intima when compared with that in vessels with a limited intimal cushion (white bars; ${ }^{*} P<.03$ ). In vessels with TVD (B; black bars), there is significantly more granzyme B in the deep intima compared with native arteries (white bars; ${ }^{*} P<.03$ ), but there is no difference in the amount of granzyme B in the media or superficial intima.

zyme B. Consistent with previous reports, staining for granzyme B was localized to leukocytes in the red pulp but was absent in the white pulp (Fig. 1A) (18). There was no staining when normal goat IgG was used in the place of the primary antibody.
In mildly diseased native vessels, there was very weak to no granzyme B positivity. Faint, diffuse staining was observed in some fatty streaks (Fig. 1B). In vessels with advanced atherosclerotic disease, granzyme B staining was very prominent and 


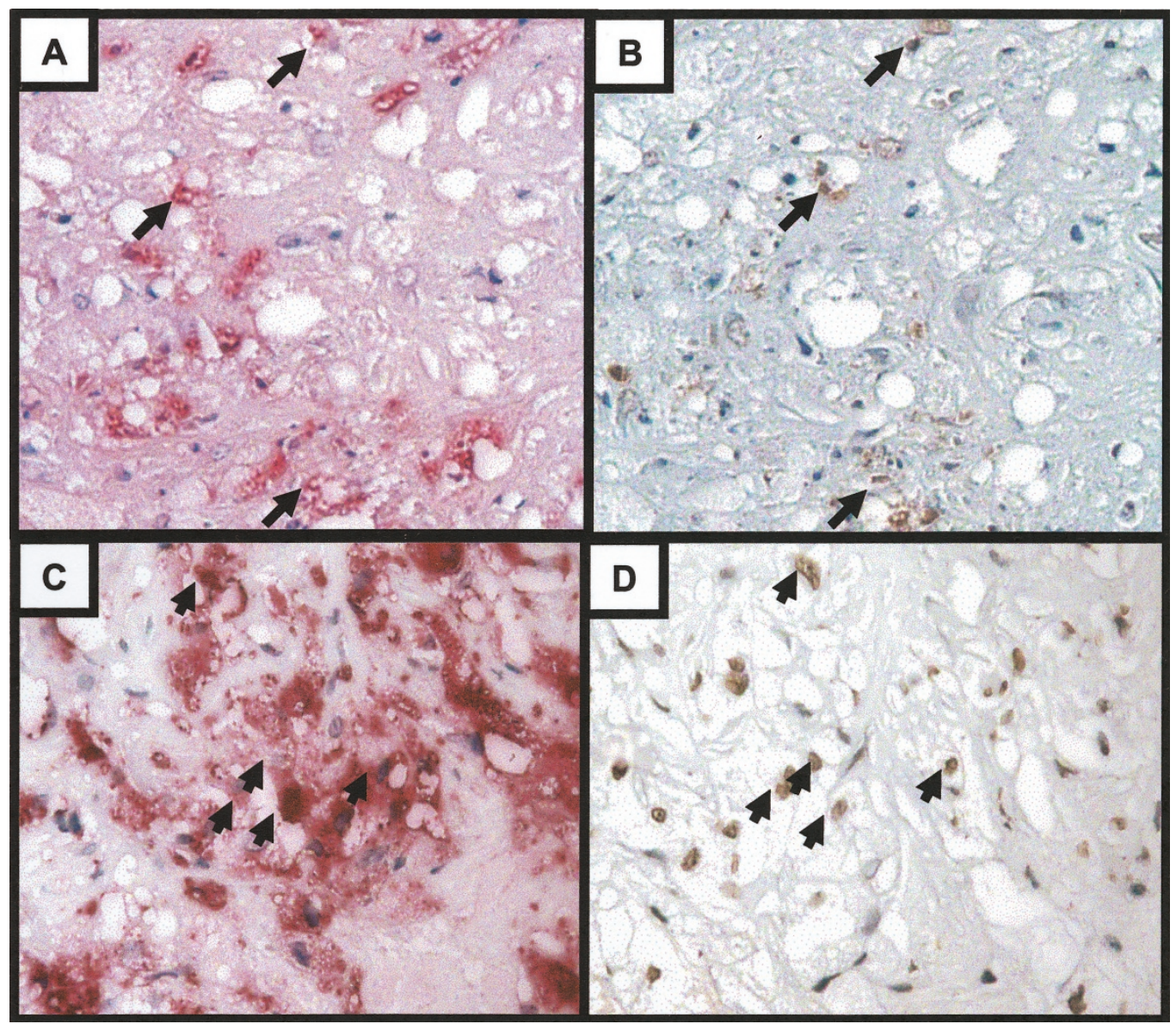

FIGURE 4. Granzyme B localizes to TUNEL-positive foam cells. Serial sections from vessels with advanced atherosclerosis and TVD were stained with anti-granzyme B (red color) and TUNEL (brown color). In advanced atherosclerotic lesions, granzyme B (A) strongly localizes to TUNELpositive foam cells (B) in the intima (arrows; $320 \times$ ). In vessels with TVD, a similar localization of granzyme B (C) to TUNEL-positive foam cells (D) is observed in the intima (arrows; $320 \times$ ).

multifocal and was observed in all regions of the vessel. There was frequent staining of deep intimal cells. Intense staining was observed in lipid-rich areas and was greatest in cellular regions surrounding the atheromatous core. Granzyme B was observed to accumulate both underneath and adjacent to the atheromatous core and was also localized to areas near the shoulder region of the atheroma. A large proportion of granzyme B was localized intracellularly in foam cells (Fig. 1C). There was moderate staining in the media of vessels with advanced atherosclerosis, and the majority of granzyme B staining in this region was very punctate, with apparent localization to infiltrating $\mathrm{T}$ cells. Occasional granzyme B positivity was also observed intracellularly in medial SMC (Fig. 1E). Also, some granzyme B positivity was observed in the media of small adventitial arteries (Fig. 1G). Endothelial cell positivity for granzyme B was not apparent.

In vessels with TVD, granzyme B immunoreactivity was very dramatic in the developed intima and was also observed in the media and adventitia. Granzyme B positivity in the deep intima was very focal, often localizing to foam cells surrounding lipid-rich regions (Fig. 1D). In the media of some vessels, granzyme B positivity appeared to localize intracellularly in several SMC (Fig. 1F). In the adventitia, granzyme B localized to leukocytes in inflammatory infiltrates as well as to the media of some adventitial arteries (Fig. $1 \mathrm{H}$ ).

To ensure the specificity of the polyclonal antibody used to detect granzyme B in the immunohistochemical staining, a Western blot was performed using the same antibody. Granzyme B is approximately $32 \mathrm{kDa}$ in size. In samples from both atherosclerotic and TVD vessels, there was a single band of $\sim 32 \mathrm{kDa}$, indicating that the antibody used in this study is specific for granzyme B (Fig. 2).

Granzyme B positivity in the media, deep intima, and superficial intima was assessed semiquantitatively in both diseases. In all areas of vessels with advanced atherosclerosis, there was significantly more granzyme B as compared with vessels with mild disease (Fig. 3A). In vessels with TVD, there was significantly more granzyme B in the deep intima than in native arteries with mild disease (Fig. 3B). Unlike the advanced atherosclerotic cases, 


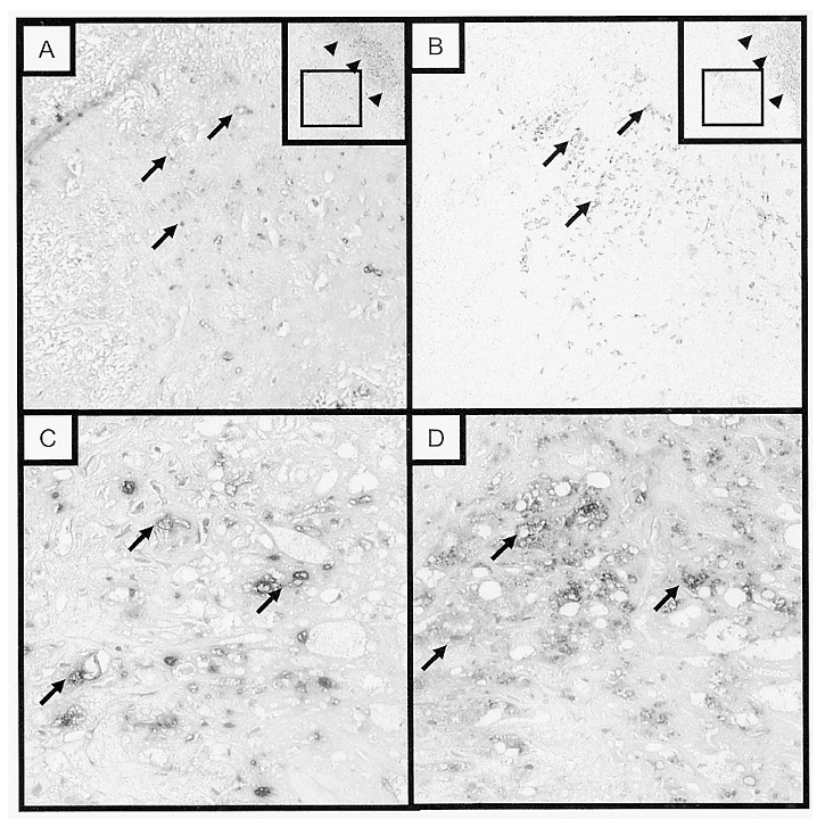

FIGURE 5. Serial sections from vessels with advanced atherosclerosis were stained for granzyme B (A) and SMC $\alpha$-actin (B). Insets are lower power micrographs of serial granzyme B-positive and SMC

$\alpha$-actin-positive regions of advanced atherosclerotic arteries. Granzyme B localizes to smooth muscle foam cells in the intima underlying and surrounding the atheromatous core (arrows; $320 \times$, insets, $160 \times$ ). Serial sections from vessels with advanced atherosclerosis were also stained for granzyme B (C) and CD68 (D). In the shoulder regions of the plaque, granzyme B localizes to macrophages (arrows; $320 \times$ ).

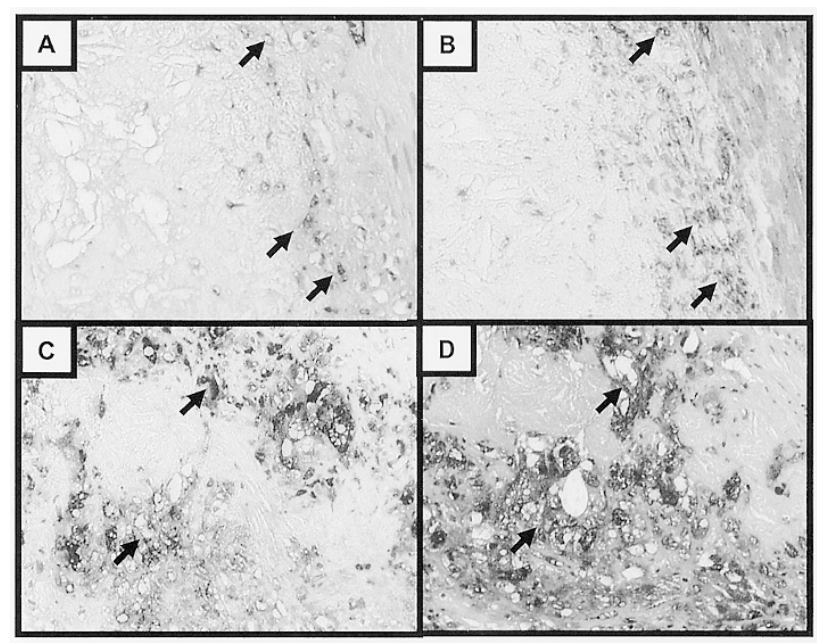

FIGURE 6. Serial sections from vessels with TVD were stained for granzyme B (A) and SMC $\alpha$-actin (B). Granzyme B localizes to smooth muscle foam cells in the intima underlying and surrounding the atheromatous core (arrows; $320 \times$, insets $160 \times$ ). Serial sections from vessels with TVD were also stained for granzyme B (C) and CD68 (D). In lipid-rich areas of the plaque, granzyme B localizes to macrophages (arrows; $320 \times$ ). there was no significant difference in the amount of granzyme B in the media and superficial intima of TVD vessels when compared with native vessels with mild disease. This increased abundance of granzyme B only in the deep intima may be due to the localization of lipid-rich regions primarily in the deep intima of the studied TVD lesions.

\section{Granzyme B Localizes to TUNEL-Positive Cells}

The presence of apoptotic cells in the intima of diseased vessels was assessed using TUNEL staining. In all cases, TUNEL positivity in intimal cells was very localized and specific to cellular nuclei, suggesting an intact nuclear membrane reminiscent of apoptosis as compared with necrosis (Fig. 4). Staining of serial sections from vessels with advanced atherosclerosis and TVD with anti-granzyme B and TUNEL revealed that many granzyme B-positive foam cells surrounding atheromatous areas were TUNEL positive (Fig. 4).

\section{Granzyme B Localizes to SMC and Macrophages}

Staining of serial sections with anti-granzyme B and anti-smooth muscle $\alpha$-actin or anti-CD68 revealed that granzyme B accumulated in both SMC and macrophages in advanced atherosclerosis (Fig. 5) and TVD (Fig. 6). In both diseases, localization of granzyme B SMC was greatest in the deep intima and in lipid-rich regions around the atheroma, whereas localization to macrophages was mostly in the atheromatous core and in the shoulder region of atherosclerotic plaques.

\section{Granzyme B Localizes to T Cells}

Serial sections were stained for granzyme B and CD3 to determine whether granzyme B localized to $\mathrm{T}$ cells in the intima of diseased vessels. In atherosclerotic lesions, CD3 staining was prominent, especially surrounding and within the atheroma. In T-cell infiltrates, many CD3-positive cells were also granzyme B positive (Fig. 7). A similar pattern of staining was observed in TVD cases. In the intima of these vessels, several CD3-positive cells were also granzyme B positive (Fig. 7).

\section{Granzyme B mRNA Localizes to Infiltrating Leukocytes in TVD}

In situ hybridization for granzyme B mRNA was performed to determine the types of cells express-

FIGURE 8. In situ hybridization was performed on vessels with TVD to identify the types of cells expressing granzyme B. Tonsil was used as our control tissue (A). Consistent with previous reports, granzyme B mRNA positivity (red color; arrows) is observed in the interfollicular space but is absent from the cortex of the follicles ( $\mathrm{I}=$ interfollicular space, $\mathrm{F}=$ follicle; $120 \times$ ). There was no staining in sections stained with an irrelevant probe (antisense to coxsackievirus RNA; bottom right inset; $120 \times$ ). In the area underlying the endothelium (B), in the deep intima (C), and in the adventitia (D) of vessels with TVD, granzyme B mRNA was localized to small, infiltrating mononuclear cells (arrows; $320 \times$ ). The bottom left insets are photomicrographs of the same area of the corresponding figure in which a color overlay (yellow color) has been applied to positive staining that was detected using a color segmentation file developed in ImageProPlus. No granzyme B mRNA was observed in foam cells surrounding lipid-rich areas of the intima. 


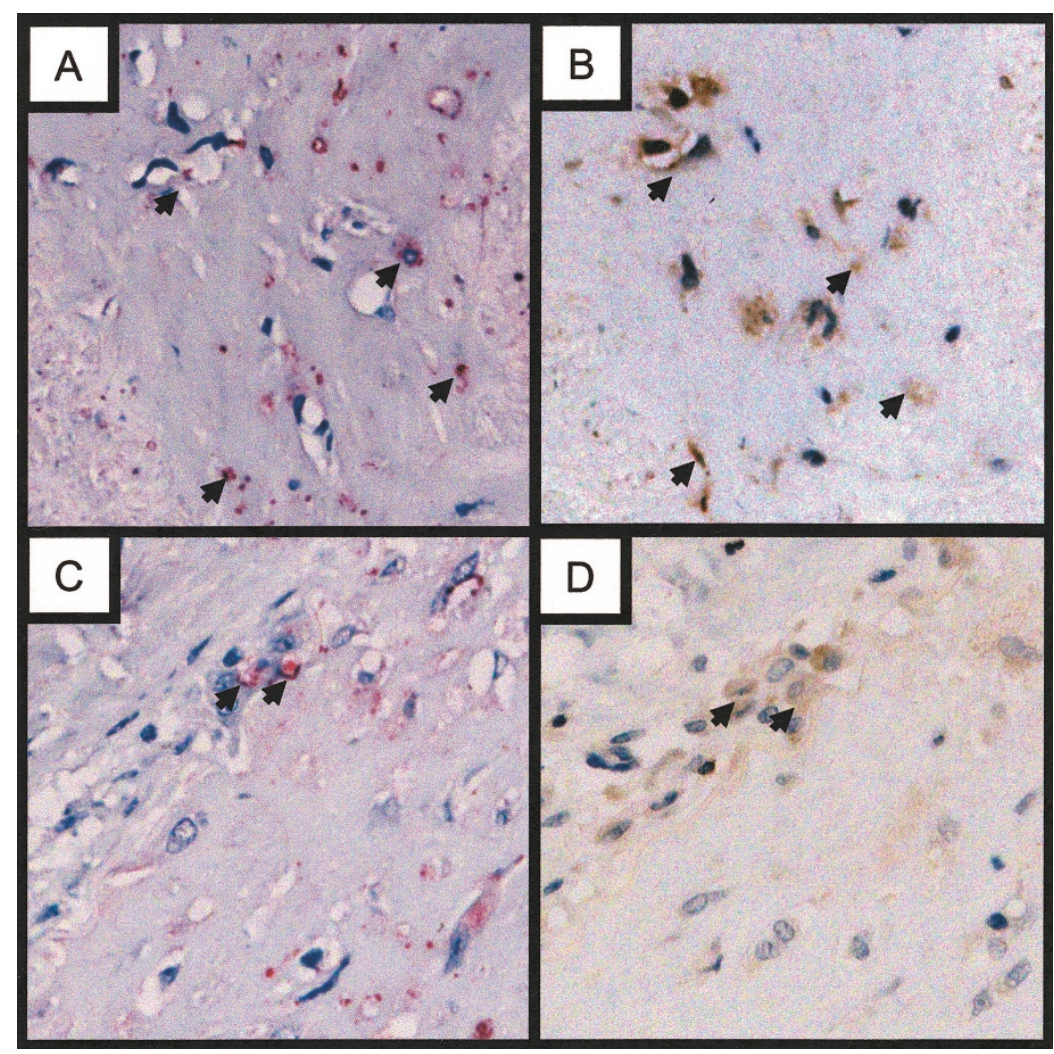

FIGURE 7. Serial sections from vessels with atherosclerosis and TVD were stained for granzyme B and CD3. In vessels with atherosclerosis, granzyme B (A; red color) localizes to some T cells (B; brown color) in the intima (arrows; $320 \times)$. In the intima of vessels with TVD, granzyme B (C) localizes to $\mathrm{T}$ cells $(\mathbf{D}$; arrows; $320 \times)$.

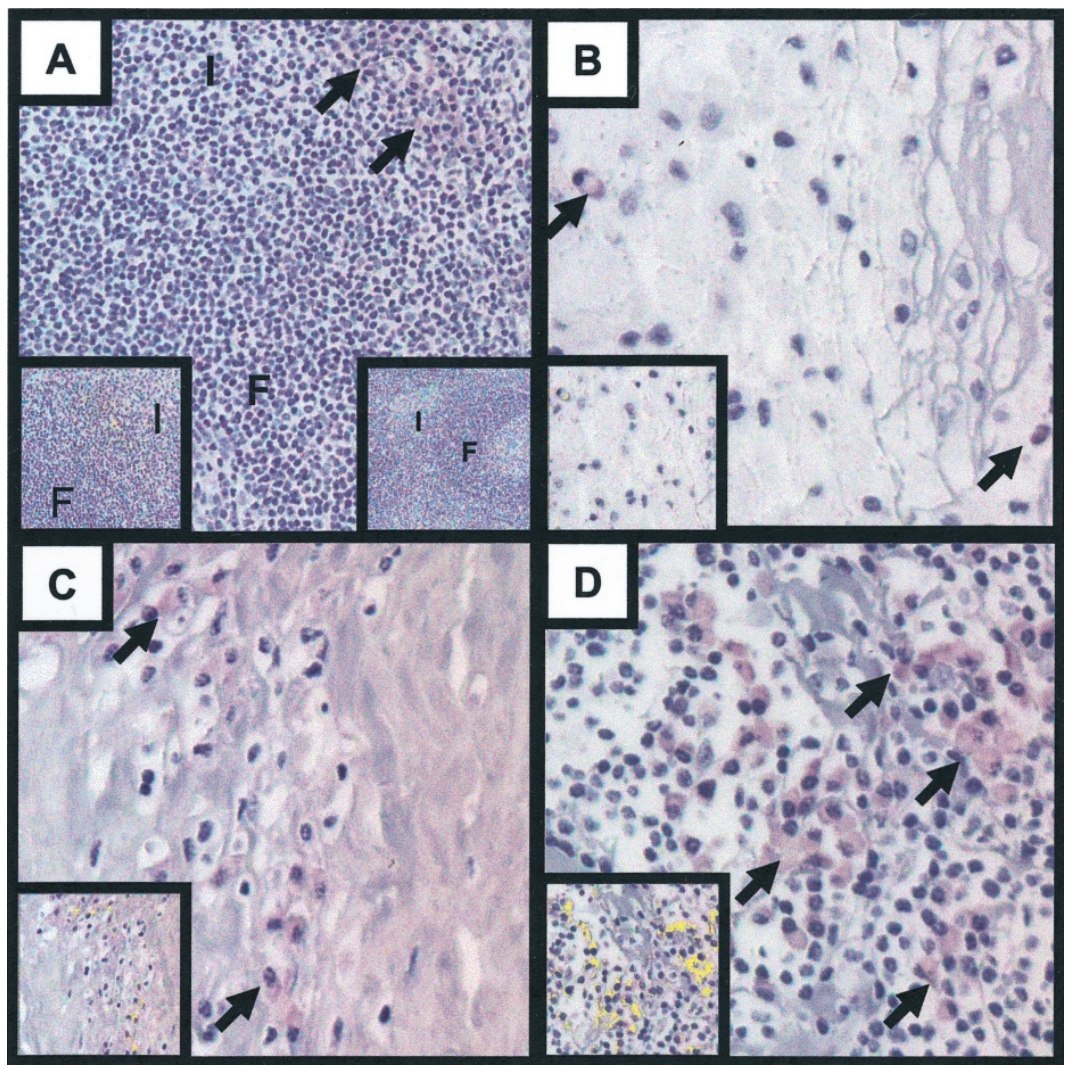


ing granzyme B. Sections from human tonsil were used to optimize this technique. Consistent with previous reports, granzyme B mRNA in the tonsil localized to leukocytes in the interfollicular space but was absent from the cortex of follicles (Fig. 8A) (18). No vessels with advanced atherosclerosis contained sufficient levels of RNA for this procedure (as determined by acridine/orange staining). However, RNA levels in vessels with TVD were well preserved. In these vessels, there was no granzyme B mRNA in foam cells, suggesting that these cells are not expressing this protease. Granzyme B mRNA in TVD lesions localized primarily to small, infiltrating inflammatory cells in the intima and adventitia (Fig 8B-D). Many of these cells were located in the area underlying the endothelium, in the deep intima, and in perivascular infiltrates in the adventitia. All are regions in which there is marked immune cell infiltration. Further, the size and shape of these cells suggest that they may be infiltrating lymphocytes.

\section{DISCUSSION}

In the current report, we characterize the presence of granzyme B in atherosclerotic and TVD lesions and associate the presence of this protease with increasing atherosclerotic disease severity. Granzyme B localization was very similar in both atherosclerosis and TVD. It was observed predominantly in and around atheromatous regions, whereupon it localized to TUNEL-positive foam cells, suggesting that granzyme B could be contributing, at least in part, to cell death in areas surrounding lipid-rich regions. Through these effects, granzyme B may contribute to growth of atheromas. We further characterize the localization of granzyme B to intimal SMC, macrophages, and T cells, and identify infiltrating leukocytes as a source of this cytotoxic protease in TVD.

In atherosclerosis, immune-mediated cytotoxicity in the intima may be an important event in restructuring of the atherosclerotic plaque from a stable to an unstable, rupture-prone lesion $(19,20)$. Indeed, FasL localizes to apoptotic SMC in advanced lesions and induces apoptosis of SMC in vitro $(21,22)$. In addition, the cellular effectors of immune-mediated apoptosis in atherosclerotic plaques are likely $\mathrm{T}$ cells and macrophages, as these leukocytes have been implicated in SMC apoptosis and in the development of acute coronary syndromes $(8,23-27)$. Our association of granzyme B immunoreactivity with advanced atherosclerotic disease, and localization of granzyme B to apoptotic cells, suggests that this cytotoxic immune factor may further contribute to acute coronary syndromes through a similar mechanism as FasL. Al- ternatively, because granzyme B proteolysis has been implicated in the generation of autoantigens in certain autoimmune diseases, granzyme B activity in atheromatous lesions could also lead to the generation of autoantigens in affected vessels (28).

The mechanisms and potential consequences of smooth muscle cell apoptosis in the intima of TVD lesions have not been well studied. Our group has previously shown that increased lipid accumulation and deposition within the intima of TVD may promote lesion severity (29-31). Specifically, increased luminal narrowing is correlated with increased lipid deposition in TVD (30). Because we have shown that granzyme B localizes to apoptotic foam cells surrounding lipid-rich regions, this form of immunemediated cytotoxicity may contribute to growth of the acellular atheroma and lipid deposition by reducing the lipid-clearing ability of intimal cells. Further, previous reports in knockout mice have shown that certain aspects of the immune response, although not decreasing overall intimal thickening, can alter lesion morphology from an acellular to a cell-rich lesion (32). The sequelae of this type of lesion remodeling in TVD remain obscure.

The localization of granzyme B to foam cells in atherosclerosis does not necessarily indicate that these cells are expressing this secretory granuleassociated protease. Instead, Nakajima et al. (33) have recently shown that a subset of CD4+ T cells that are abundant in patients with atherosclerosis express perforin and that these are potent mediators of granule-mediated apoptosis of endothelial cells. The expression of perforin by this subset of $\mathrm{T}$ cells is strongly correlated with unstable angina, suggesting a key role for the granzyme/perforin pathway in acute coronary syndromes. Further, granzyme $\mathrm{B}$ protein did localize to infiltrating $\mathrm{T}$ cells in the intima, suggesting that these immune infiltrates are likely expressing this protease. Detection of granzyme B mRNA expression with in situ hybridization was not possible on arteries with atherosclerosis because of low levels of RNA. This is likely caused by delayed fixation of these autopsy tissues, commonly encountered in clinical samples. However, because proteins are much more stable than RNA, our sections were acceptable for immunohistochemistry. Such is evident by the expected staining pattern of SMC $\alpha$-actin and CD68. In our advanced atherosclerotic cases, there was marked SMC $\alpha$-actin positivity in the media and in large cells in the intima, and CD68 staining in these sections was localized predominantly to lipid-rich regions in the intima.

In situ hybridization in vessels with TVD showed that granzyme B mRNA was isolated to infiltrating leukocytes in the adventitia and intima and was likely not being expressed by foam cells. Because the pattern and localization of granzyme B in vessels with 
TVD was very similar to that in vessels with atherosclerosis, the source of granzyme B in advanced atherosclerosis could be the same as in TVD, namely infiltrating leukocytes. In support of this notion, although some pathological aspects of atherosclerosis and moderate-severe TVD are distinct, the contribution of the T-cell-mediated response to both diseases may be similar in many respects. For instance, both are inflammatory disorders and there is a specific T-cell-generated immune response to certain antigens in both atherosclerosis and TVD that contribute to vascular damage $(34,35)$. In atherosclerosis, antigen-activated T-cell responses are generated toward oxLDL, resulting in increased atherogenesis and plaque degeneration $(7,36,37)$. In TVD, a similar response is generated toward several foreign epitopes in the allograft. These events contribute to the underlying vascular damage, resulting in the accumulation of lipids and oxLDL and the potential for an immune response toward oxLDL in established lesions (30, 38, 39). Finally, CD8 + and CD4 $+\mathrm{T}$ cells accumulate in the intima of both diseases, and FasL is expressed by both $\mathrm{T}$ cells and macrophages in both atherosclerosis and TVD. This similarity in FasL expression suggests a similar mechanism of cytotoxic immune damage to the vasculature in both diseases $(8,25,40)$.

In summary, we have localized granzyme B to advanced atherosclerotic and TVD lesions, and associated increased granzyme B with atherosclerotic disease severity. Further, we have shown the localization of this apoptotic protease to foam cells, many of which are undergoing cellular damage suggestive of apoptosis. These data suggest that granzyme B may be responsible, at least in part, for the induction of cell death in smooth muscle and macrophage-derived foam cells surrounding atheromas. To our knowledge, this is the first characterization of granzyme B in atherosclerotic and TVD lesions.

Acknowledgments: We are very grateful to $D r$. R. Chris Bleackley for the kind gift of granzyme B cDNA.

\section{REFERENCES}

1. Pollman MJ, Hall JL, Mann MJ, Zhang L, Gibbons GH. Inhibition of neointimal cell bcl-x expression induces apoptosis and regression of vascular disease. Nat Med 1998;4:222-7.

2. Geng YJ, Libby P. Evidence for apoptosis in advanced human atheroma. Colocalization with interleukin-1 beta-converting enzyme. Am J Pathol 1995;147:251-66.

3. von der Thusen JH, van Vlijmen BJ, Hoeben RC, Kockx MM, Havekes LM, van Berkel TJ, et al. Induction of atherosclerotic plaque rupture in apolipoprotein $\mathrm{E}-1$ - mice after adenovirusmediated transfer of p53. Circulation 2002;105:2064-70.

4. Choy JC, Granville DJ, Hunt DW, McManus BM. Endothelial cell apoptosis: biochemical characteristics and potential implications for atherosclerosis. J Mol Cell Cardiol 2001;33:1673-90.

5. Hruban RH, Beschorner WE, Baumgartner WA, Augustine SM, Ren H, Reitz BA, et al. Accelerated arteriosclerosis in heart transplant recipients is associated with a T-lymphocytemediated endothelialitis. Am J Pathol 1990;137:871-82.

6. Zhou X, Nicoletti A, Elhage R, Hansson GK. Transfer of CD4(+) $\mathrm{T}$ cells aggravates atherosclerosis in immunodeficient apolipoprotein E knockout mice. Circulation 2000;102:2919-22.

7. Caligiuri G, Paulsson G, Nicoletti A, Maseri A, Hansson GK. Evidence for antigen-driven T-cell response in unstable angina. Circulation 2000;102:1114-9.

8. Boyle JJ. Association of coronary plaque rupture and atherosclerotic inflammation. J Pathol 1997;181:93-9.

9. Granville DJ, Carthy CM, Hunt DW, McManus BM. Apoptosis. Molecular aspects of cell death and disease. Lab Invest 1998;78:893-913.

10. Trapani JA, Davis J, Sutton VR, Smyth MJ. Proapoptotic functions of cytotoxic lymphocyte granule constituents in vitro and in vivo. Curr Opin Immunol 2000;12:323-9.

11. Bleackley RC, Heibein JA. Enzymatic control of apoptosis. Nat Prod Rep 2001;18:431-40.

12. Jacob T, Ascher E, Hingorani A, Khandros Y, Tsemekhin B, Zeien L, et al. Differential proteolytic activity and induction of apoptosis in fibrous versus atheromatous plaques in carotid atherosclerotic disease. J Vasc Surg 2001;33:614-20.

13. Dong C, Wilson JE, Winters GL, McManus BM. Human transplant coronary artery disease: pathological evidence for Fas-mediated apoptotic cytotoxicity in allograft arteriopathy. Lab Invest 1996;74:921-31.

14. Lin H, Wilson JE, Roberts CR, Horley KJ, Winters GL, Costanzo MR, et al. Biglycan, decorin, and versican protein expression patterns in coronary arteriopathy of human cardiac allograft: distinctness as compared to native atherosclerosis. J Heart Lung Transplant 1996;15:1233-47.

15. Granville DJ, Shaw JR, Leong S, Carthy CM, Margaron P, Hunt DW, et al. Release of cytochrome c, Bax migration, Bid cleavage, and activation of caspases 2, 3, 6, 7, 8, and 9 during endothelial cell apoptosis. Am J Pathol 1999;155:1021-5.

16. Granville DJ, Cassidy BA, Ruehlmann DO, Choy JC, Brenner C, Kroemer G, et al. Mitochondrial release of apoptosisinducing factor and cytochrome c during smooth muscle cell apoptosis. Am J Pathol 2001;159:305-11.

17. Kanda T, Utsugi T, Kawazu S, Wilson JE, Yang D, Suarez A, et $a l$. Induction of virus-induced IDDM in virus resistant mice without lymphocyte maturation. Life Sci 1998;63:33-40.

18. Kummer JA, Wever PC, Kamp AM, ten Berge IJ, Hack CE, Weening JJ. Expression of granzyme A and B proteins by cytotoxic lymphocytes involved in acute renal allograft rejection. Kidney Int 1995;47:70-7.

19. Neri Serneri GG, Prisco D, Martini F, Gori AM, Brunelli T, Poggesi L, et al. Acute T-cell activation is detectable in unstable angina. Circulation 1997;95:1806-12.

20. Shah PK. Plaque disruption and coronary thrombosis: new insight into pathogenesis and prevention. Clin Cardiol 1997; 20:II-38-44.

21. Esaki T, Hayashi T, Muto E, Kano H, Kumar TN, Asai Y, et al. Expression of inducible nitric oxide synthase and Fas/Fas ligand correlates with the incidence of apoptotic cell death in atheromatous plaques of human coronary arteries. Nitric Oxide 2000;4:561-71.

22. Sata M, Suhara T, Walsh K. Vascular endothelial cells and smooth muscle cells differ in expression of Fas and Fas ligand and in sensitivity to Fas ligand-induced cell death: implications for vascular disease and therapy. Arterioscler Thromb Vasc Biol 2000;20:309-16.

23. Bjorkerud S, Bjorkerud B. Apoptosis is abundant in human atherosclerotic lesions, especially in inflammatory cells (macrophages and T cells), and may contribute to the accumulation of gruel and plaque instability. Am J Pathol 1996; 149:367-80.

24. Seshiah PN, Kereiakes DJ, Vasudevan SS, Lopes N, Su BY, Flavahan NA, et al. Activated monocytes induce smooth 
muscle cell death: Role of macrophage colony-stimulating factor and cell contact. Circulation 2002;105:174-80.

25. Boyle JJ, Bowyer DE, Weissberg PL, Bennett MR. Human blood-derived macrophages induce apoptosis in human plaque-derived vascular smooth muscle cells by Fas-ligand/ Fas interactions. Arterioscler Thromb Vasc Biol 2001;21:1402-7.

26. Perlman H, Pagliari LJ, Georganas C, Mano T, Walsh K, Pope RM. FLICE-inhibitory protein expression during macrophage differentiation confers resistance to fas-mediated apoptosis. J Exp Med 1999;190:1679-88.

27. Yao PM, Tabas I. Free cholesterol loading of macrophages induces apoptosis involving the fas pathway. J Biol Chem 2000;275:23807-13.

28. Gahring L, Carlson NG, Meyer EL, Rogers SW. Granzyme B proteolysis of a neuronal glutamate receptor generates an autoantigen and is modulated by glycosylation. J Immunol 2001;166:1433-8.

29. McManus BM, Malcom G, Kendall TJ, Gulizia JM, Wilson JE, Winters G, et al. Lipid overload and proteoglycan expression in chronic rejection of the human transplanted heart. Clin Transplant 1994;8:336-40.

30. McManus BM, Horley KJ, Wilson JE, Malcom GT, Kendall TJ, Miles RR, et al. Prominence of coronary arterial wall lipids in human heart allografts. Implications for pathogenesis of allograft arteriopathy. Am J Pathol 1995;147:293-308.

31. Wilson JE, Wood S, McDonald P, Kenyon J, Dong C, McManus BM. Contribution of lipids to the pathogenesis of transplant vascular sclerosis. Transpl Immunol 1997;5:247-50.
32. Russell PS, Chase CM, Colvin RB. Alloantibody- and T. cellmediated immunity in the pathogenesis of transplant arteriosclerosis: lack of progression to sclerotic lesions in B celldeficient mice. Transplantation 1997;64:1531-6.

33. Nakajima T, Schulte S, Warrington KJ, Kopecky SL, Frye RL, Goronzy JJ, et al. T-cell-mediated lysis of endothelial cells in acute coronary syndromes. Circulation 2002;105:570-5.

34. Ross R. Atherosclerosis is an inflammatory disease. Am Heart J 1999;138:S419-20.

35. McDonald PC, Wong D, Granville DJ, McManus BM. Emerging roles of endothelial cells and smooth muscle cells in transplant vascular disease. Transplant Rev 1999;13:1-19.

36. Laurat E, Poirier B, Tupin E, Caligiuri G, Hansson GK, Bariety $\mathrm{J}$, et al. In vivo downregulation of $\mathrm{T}$ helper cell 1 immune responses reduces atherogenesis in apolipoprotein E-knockout mice. Circulation 2001;104:197-202.

37. Zhou X, Caligiuri G, Hamsten A, Lefvert AK, Hansson GK. LDL immunization induces T-cell-dependent antibody formation and protection against atherosclerosis. Arterioscler Thromb Vasc Biol 2001;21:108-14.

38. Miller LW, Granville DJ, Narula J, McManus BM. Apoptosis in cardiac transplant rejection. Cardiol Clin 2001;19:14154.

39. McDonald PC, Kenyon JA, McManus BM. The role of lipids in transplant vascular disease. Lab Invest 1998;78:1187201.

40. Xu B, Sakkas LI, Slachta CA, Goldman BI, Jeevanandam V, Oleszak EL, et al. Apoptosis in chronic rejection of human cardiac allografts. Transplantation 2001;71:1137-46. 